\title{
Sopro Cardíaco Pediátrico: estudo de série de casos
}

Rita Oliveira, ${ }^{1}$ Luís Martins, ${ }^{2}$ Helena Andrade, ${ }^{3}$ António Pires, ${ }^{4}$ Eduardo Castela ${ }^{5}$

\section{RESUMO}

Introdução: Em cerca de metade da população pediátrica é detectado um sopro cardíaco (SC) em algum momento da sua vida. Os SC constituem uma causa frequente de referenciação a Consulta de Pediatria e/ou Cardiologia Pediátrica, pelo que se torna importante o conhecimento dos sinais de alarme evocadores de cardiopatia na avaliação de uma criança com SC.

Objectivo: Estudo da população referenciada a Consulta de Cardiologia Pediátrica entre Janeiro e Março de 2012 com o diagnóstico de SC. Material e Métodos: Estudo de uma série de casos de crianças referenciadas por SC. Foram analisados dados, avaliando variáveis demográficas, anamnésicos, sinais clínicos e resultado dos exames complementares, nomeadamente ecocardiografia transtorácica e electrocardiograma.

Resultados: No período em análise, realizaram-se um total de 743 primeiras consultas de Cardiologia Pediátrica, das quais em 197 (26,5\%) o motivo de referenciação foi SC. Das crianças avaliadas, 51,3\% pertenciam ao sexo masculino e a maioria (cerca de $70 \%$ ) tinha idade inferior a 2 anos. Na quase totalidade das crianças o SC apresentava características de sopro inocente e o restante exame objectivo não apresentava alterações de relevo. Em cerca de $1 / 3$ dos casos foi detectada alteração na avaliação ecocardiográfica. Do cruzamento entre as diferentes variáveis em análise destaca-se uma relação estatisticamente significativa entre auscultação de sopro não inocente, alterações ao exame objectivo e ecocardiografia patológica. Verificou-se também uma associação positiva e com significância estatística entre o grupo etário inferior a 1 ano e os achados ecocardiográficos.

Discussão: O SC em idade pediátrica é um motivo frequente de referenciação a Consulta da especialidade. Na sua maioria, o SC em pediatria é uma condição benigna e as características clínicas parecem suficientes para diferenciação de uma condição potencialmente patológica. Tal como descrito em estudos anteriores, alterações ao exame físico e idade inferior a 2 anos são sinais de alarme que requerem avaliação mais detalhada.

Palavras-chave: Sopro Cardiaco; Pediatria.

\section{INTRODUÇÃO}

U m sopro cardíaco (SC) corresponde a um som extra, audível para além dos normais tons cardíacos, que surge em resultado de uma turbulência no fluxo sanguíneo capaz de gerar vibrações de intensidade suficiente para serem transmitidas através da parede torácica. ${ }^{1}$

Constitui um importante elemento diagnóstico na detecção de uma cardiopatia congénita, permitindo avaliar,

${ }^{1}$ Interna Complementar de Pediatria - Hospital São Teotónio, Centro Hospitalar Tondela-Viseu

'Interno Complementar de Pediatria - Hospital Pediátrico Carmona da Mota, Centro Hospitalar e Universitário de Coimbra

${ }^{3}$ Assistente Hospitalar de Cardiologia Pediátrica - Hospital Pediátrico Carmona da Mota, Centro Hospitalar e Universitário de Coimbra

${ }^{4}$ Assistente Hospitalar Graduado de Cardiologia Pediátrica - Hospital Pediátrico Carmona da Mota, Centro Hospitalar e Universitário de Coimbra

${ }^{5}$ Chefe de Serviço de Cardiologia Pediátrica - Hospital Pediátrico Carmona da Mota, Centro Hospitalar e Universitário de Coimbra ainda que grosseiramente, a sua gravidade, bem como vigiar o seu curso clínico.

São vários os factores que intervêm na produção de um SC: o aumento de volume da corrente sanguínea, a passagem do fluxo sanguíneo através de uma estrutura valvular anormal, cavidade/vaso dilatado ou hipertrofiado ou, ainda, a regurgitação do fluxo sanguíneo em válvulas incompetentes ou defeitos congénitos.

No período neonatal, a prevalência do SC situa-se entre 0,6 a $4,2 \%$ da população nesta faixa etária; após este período, estima-se que cerca de $90 \%$ das crianças terão um SC detectável durante a sua infância. . $^{2,3}$ Em aproximadamente 50 a $70 \%$ das crianças em idade escolar, élhes identificado um SC durante uma observação médica de rotina, ${ }^{3}$ apresentando um pico máximo de incidência aos 5 anos, a qual diminui drasticamente após os 10 anos de idade. ${ }^{1}$

Em idade pediátrica, poderão definir-se três tipos 
principais de SC:

- o sopro inocente, quando ocorre num coração estrutural e funcionalmente normal;

- o sopro funcional ou fisiológico se, embora na ausência de anomalia cardiovascular, existe uma alteração hemodinâmica que concorre para a alteração do normal fluxo sanguíneo (como por exemplo as situações de anemia, hipertiroidismo, intercorrências febris e, numa percentagem significativa de crianças, após um esforço físico moderado a intenso);

- por último, o sopro patológico ou orgânico, que decorre da presença de anomalias estruturais e funcionais do sistema cardiovascular.

A diferenciação entre um sopro inocente e um sopro patológico em geral é dada pelas suas características clínicas comuns: é considerado um sopro patológico ou orgânico aquele que apresenta uma intensidade na auscultação superior ao grau II/VI da escala de Levine, com irradiação cervical ou ao dorso, fixo, meso ou pansistólico ou aquele audível (também) na diástole.

O sopro patológico em idade pediátrica é causado maioritariamente por alterações valvulares ou defeitos septais; no entanto, outras cardiopatias podem estar presentes, quer do foro congénito (persistência do canal arterial ou coartação da aorta) quer adquiridas (exemplo da cardiomiopatia dilatada). ${ }^{7}$

Apesar da sua elevada frequência e do facto de a maioria dos SC em idade pediátrica não serem patológicos, podem ser a única manifestação de uma cardiopatia, ${ }^{4,5}$ cerca de $70 \%$ dos SC em crianças e adolescentes são assintomáticos. ${ }^{3,8,9}$

Assim, na prática clínica a auscultação de um SC em idade pediátrica pode constituir um verdadeiro dilema diagnóstico e, dado o potencial de gravidade que lhes poderá estar subjacente, a sua detecção condiciona, por norma, a referenciação a consulta de sub-especialidade, ainda que sinais clínicos indicadores de ausência de benignidade sejam detectados.

\section{OBJECTIVOS}

Com este trabalho pretendemos caracterizar a população referenciada a Consulta de Cardiologia Pediátrica de um Centro Hospitalar Terciário por SC, analisando a existência de factores associados a sopro patológico, nomeadamente no que diz respeito a aspectos demográficos, sinais clínicos ou achados no exame objectivo.

\section{MATERIAL E MÉTODOS}

O presente estudo é um estudo descritivo de série de casos.

Para a realização do presente trabalho foi utilizada uma amostra populacional de conveniência, constituída por crianças ou adolescentes observados em Consulta de Cardiologia Pediátrica do Hospital Pediátrico Carmona da Mota (HPCM) entre o período de 1 de Janeiro e 31 de Março de 2012, inclusive, com motivo de consulta SC.

Definiram-se como critérios de inclusão o motivo de referenciação por SC, não se excluindo qualquer caso.

Definiu-se com SC inocente aquele que apresentava as seguintes características: SC que ocorre no início da sístole (protosistólico), é de curta duração e de baixa intensidade (grau I ou II/VI da escala de Levine), tem qualidade musical ou vibrante e não apresenta irradiação para localizações fora da região precordial; alteração da intensidade com as mudanças de posição da criança.

Um SC foi classificado como patológico se apresenta uma intensidade na auscultação superior ao grau II/VI da escala de Levine, fixo, com irradiação cervical ou ao dorso, meso ou pansistólico ou aquele audível na diástole.

Procedeu-se à consulta retrospectiva do processo clínico informático das crianças referenciadas por SC, avaliando variáveis demográficas, dados anamnésicos, sinais clínicos, descrição do exame objectivo e resultado dos exames complementares, nomeadamente ecocardiografia transtorácica e electrocardiograma.

O tratamento estatístico dos dados foi realizado através do Microsoft Excel® e da aplicação SPSS® versão 15.0, realizando-se uma avaliação estatística descritiva e análise entre as diferentes variáveis através da aplicação do teste de qui-quadrado, definindo-se resultados com significância estatística se $\mathrm{p}<0,05$.

\section{RESULTADOS}

No período em análise, realizaram-se um total de 743 primeiras consultas de Cardiologia Pediátrica e, em 197 casos (26,5\%), o motivo de referenciação foi SC.

Da amostra final de crianças avaliadas com o diagnóstico de SC, 101 (53,1\%) pertenciam ao sexo masculino; no que diz respeito à idade, a série em análise apresentava uma idade média à data da consulta de 21 meses com um desvio padrão de 13 meses, sendo que a maioria (sensivelmente $70 \%$ do total) incluía-se na faixa etária inferior a 24 meses.

Da avaliação das características auscultatórias, na maio- 
ria dos casos (109, cerca de 96\%) o SC apresentava características inocentes. Em apenas 7 crianças $(3,6 \%)$ inferia-se o diagnóstico de sopro patológico e em 4 destes 7 casos foram encontradas outras alterações ao exame objectivo que pudessem corroborar a hipótese de doença cardiovascular, mormente sinais de dificuldade respiratória, hepatomegália e má evolução estaturo-ponderal.

Da avaliação por exames complementares de diagnóstico, mormente o electrocardiograma, verificamos que este foi efectuado na totalidade dos casos observados, encontrando-se um caso de extrassistolia supraventricular e um caso de bloqueio auriculo-ventricular de $2 .{ }^{\circ}$ grau em crianças com sopro de características inocentes.

Quando analisados os dados relativos à realização de ecocardiograma transtorácico, também realizado em todas as crianças, foi detectada alteração estrutural ou funcional cardíaca em cerca de um terço dos casos ( $n=67$ ), sendo a alteração mais frequentemente encontrada a Comunicação interauricular (CIA) em $40 \%$ dos casos $(n=26)$, seguida da Comunicação interventricular em 15\% ( $\mathrm{n}=10$ ) (Quadro 1).

Cruzando as características do sopro com o resultado do ecocardiograma, pudemos constatar que todas as crianças que apresentavam um SC de características patológicas ou outras alterações no exame objectivo apresentaram um ecocardiograma alterado; em cerca de $32 \%$ dos casos de sopro inocente, foi igualmente detectada uma condição patológica após a realização do ecocardiograma. Salienta-se também o facto de nenhum caso de CIA, já referida anteriormente como a patologia mais frequente, apresentar um sopro de caraterísticas inocentes ou outras alterações ao exame objectivo. No Quadro 1 estão igualmente discriminadas as patologias encontradas, em função das características do sopro.

Destaca-se também uma associação estatisticamente significativa entre a idade inferior a 24 meses e alterações detectadas na ecografia cardíaca ( $<<0,001$ ) (Quadro 2). Relacionando esta faixa etária com os achados clínicos verifica-se, em 4 casos, a existência ao exame clínico de um SC orgânico/patológico, sendo que nos restantes casos em que existia um SC, este apresentava características inocentes.

Pela análise dos dados, e de acordo com o descrito no Quadro 3, a auscultação de um SC de características patológicas tem um valor preditivo positivo de doença cardíaca estrutural de 100\%; no entanto, a auscultação de um SC inocente não exclui a presença de doença cardíaca estrutural.

\section{DISCUSSÃO}

O SC em idade pediátrica é um motivo frequente de referenciação a Consulta da especialidade.

No estudo realizado inferimos uma associação positiva e estatisticamente significativa entre dados clínicos (caracteristícas do SC e outros achados ao exame objectivo compatíveis com o diagnóstico de cardiopatia) e os resultados do ecocardiograma transtorácico, confirmando uma vez mais a importância fulcral da clínica no diagnóstico de uma cardiopatia estrutural e funcional.

No que diz respeito aos exames complementares de diagnóstico, a realização de ecocardiograma transtorácico com estudo de doppler persiste como o gold standard para o diagnóstico definitivo de cardiopatias (nomeadamente cardiopatias congénitas) em doentes em idade pediátrica. ${ }^{1,8}$

Em cerca de 1/3 dos casos de SC com características de sopro inocente, o ecocardiograma foi compatível com uma cardiopatia, comprovando uma vez mais de que um SC pode ser a única manifestação. Constatamos também que, dos achados ecocardiográficos, a CIA foi a alteração mais frequentemente encontrada, indo de encontro ao descrito na literatura de que os defeitos do septo interaricular são uma das cardiopatias congénitas mais frequentes (representando cerca de 5 a $10 \%$ do total de cardiopatias de acordo com as várias séries publicadas) e que, numa percentagem significativa de casos, se apresenta clinicamente apenas como um SC, muitas vezes de características inocentes. ${ }^{2,3}$

Verificamos que as características clínicas do SC são um elemento fulcral da avaliação, uma vez que concluímos que um SC patológico é altamente específico da presença de uma cardiopatia estrutural, apresentando no nosso estudo um valor preditivo positivo de $100 \%$.

Contudo, a auscultação de um sopro inocente não exclui a presença de doença cardíaca estrutural, sendo por isso necessária a realização de ecocardiograma.

Verificamos na nossa análise que a maioria das crianças observadas tinhaidade inferior 24 meses; para esta faixa etária comprovou-se, à semelhança de estudos internacionais, uma associação relevante e com significado estatístico com a presença de alterações ecocardiográficas patológicas.

Estes dados vão de encontro aos pressupostos da American HeartAssociation, que recomenda a realização de ecocardiografia em todas as crianças com SC e idade inferior a 24 meses ainda que com SC assintomáticos, já que nesta faixa etária a probabilidade de perante um sopro inocente se 
encontrar uma cardiopatia ronda os $2 \% .^{1}$ Também no período neonatal (ou, para alguns autores, até às 6 a 8 semanas de vida) a ecocardiografia deve sempre ser realizada, uma vez que, na presença de um SC audível no exame de rotina, a probabilidade de cardiopatia aumenta de 0,6\% para $54 \%$. $^{7}$

Em contrapartida, num doente em que a ausência destes achados permitem conscientemente afirmar uma baixa probabilidade de estar perante uma patologia do foro cardíaco, o seu seguiQUADRO I. Diagnósticos obtidos após realização ecocardiograma transtorácico (n=67)

\begin{tabular}{l|c|c} 
Achado Ecocardiograma & $\begin{array}{c}\text { N. }{ }^{\circ} \text { Casos totais } \\
\text { observados }\end{array}$ & $\begin{array}{c}\text { N. }{ }^{\circ} \text { casos observados } \\
\text { com SC patológico }\end{array}$ \\
\hline Comunicação Interauricular & 26 & 0 \\
\hline Comunicação Interventricular & 10 & 2 \\
\hline Persistência Canal Arterial & 7 & 1 \\
\hline Foramen Ovale Patente & 5 & 0 \\
\hline Insuficiência Mitral & 3 & 1 \\
\hline Insuficiência Tricúspide & 3 & 0 \\
\hline Estenose Pulmonar & 2 & 0 \\
\hline Prolapso Válvula Mitral & 2 & 0 \\
\hline Válvula Aórtica bícuspide & 2 & 1 \\
\hline Insuficiência Aórtica & 2 & 1 \\
\hline Coartação Aorta & 1 & 1 \\
\hline Janela Aorto-Pulmonar & 1 & 0 \\
\hline Fístula coronária & 1 & 0 \\
\hline Dilatação da Aorta ascendente isolada & 1 & 0 \\
\hline Hipertrofia ventricular esquerda de etiologia a esclarecer & 1 & 7 \\
\hline TOTAL & 67 & \\
\hline
\end{tabular}

mento pode continuar a ser realizado pelo médico assistente, com eventual referenciação posterior se alterações patológicas surgem em avaliações seriadas ulteriores. $^{3}$

Apesar de relevante e concordante com os dados de outras séries publicadas, o estudo apresenta algumas limitações passíveis de

QUADRO II. Análise de potenciais factores de risco para ecocardiografia alterada

\begin{tabular}{l|c|c|r|r|r} 
& $\begin{array}{c}\text { Ecocardiograma } \\
\text { alterado }\end{array}$ & $\begin{array}{c}\text { Ecocardiograma } \\
\text { normal }\end{array}$ & \multicolumn{1}{c|}{$\mathbf{P}$} & \multicolumn{1}{c|}{ OR } & \multicolumn{1}{c|}{ IC } \\
\hline Sexo masculino & 37 & 64 & 0,51 & 1,27 & $0,67-2,40$ \\
\hline Idade <24 meses & 45 & 30 & $<0,0001$ & 6,82 & $3,38-13,86$ \\
\hline SC não-inocente & 7 & 0 & 0,032 & 82 & $16-98$
\end{tabular}
comprometer os resultados estatísticos, nomeadamente o facto de se tratar de uma amostra pequena, de conveniência e não aleatória, assim como o facto de se tratar de um estudo retrospectivo elaborado com base em registos clínicos anteriores, em que por vezes a descrição das características dos achados clínicos não se encontra elaborada de forma exaustiva, o que poderia comprometer a classificação dos SC

\begin{tabular}{|c|c|c|c|}
\hline & $\begin{array}{c}\text { Ecocardiograma } \\
\text { alterado }\end{array}$ & $\begin{array}{c}\text { Ecocardiograma } \\
\text { normal }\end{array}$ & Total \\
\hline SC patológico & 7 & 0 & 7 \\
\hline SC inocente & 60 & 130 & 190 \\
\hline Total & 67 & 130 & 197 \\
\hline
\end{tabular}
em inocentes ou patológicos.

Contudo, permanece como um estudo importante no sentido de reafirmar a importância da caracterização clínica do sopro cardíaca em idade pediátrica que, de acordo

com a faixa etária, é relevante na necessidade de referenciação e/ou realização de exames complementares de diagnóstico. 


\section{CONCLUSÕES}

Na sua maioria, o SC em pediatria é uma condição benigna e as características clínicas são suficientes para diferenciação de uma condição potencialmente patológica. Tal como descrito em estudos anteriores, alterações ao exame físico e idade inferior 24 meses são sinais de alarme que requerem avaliação mais detalhada, mormente por especialista em Cardiologia Pediátrica.

Afirmar, perante o doente e sua família, a benignidade de um SC inocente, sobretudo se detectado após os 2 anos de vida, é de extrema importância. $\mathrm{O}$ alerta para eventuais sinais indicadores de doença cardiovascular deve também ser tido em conta. Porque este tipo de SC sofre frequentemente alterações com a posição, bem como ao longo do crescimento e desenvolvimento, os pais e prestadores de cuidados devem ser alertados para o facto de que os SC inocentes podem desaparecer e reaparecer ao longo do curso de vida da criança.

\section{REFERÊNCIAS BIBLIOGRÁFICAS}

1. Berdejo CR. Soplo inocente: importancia diagnóstica. In: Protocolos Diagnósticos y Terapeuticos en Cardiologia Pediátrica - Protocolos AEPED 2010; 26:1-9. Disponível em: http://www.telecardiologo.com/descargas/56854.pdf [acedido em 17/12/2013].

2. Manning D, PaweletzA, Robertson JL. Management of asymptomatic heart murmurs in infants and children. Paediatr Child Health J 2009 Jan; 19 (1): $25-9$.
3. Wierwille L. Pediatric heart murmurs: evaluation and management in primary care. Nurse Pract 2011 Mar; 36 (3): 22-9.

4. Frank JE, Jacobe KM. Evaluation and management of heart murmurs in children. Am Fam Physician 2011 Oct; 84 (7): 793-800.

5. Poddar B, Basu S. Approach to a child with a heart murmur. Indian J Pediatr 2004 Jan; 71 (1): 63-6.

6. Discigil G, Aydogdu A, Gemalmaz A, Gurel FS, Basak O. Cardiac auscultatory skills of academic family physicians: strength of association with an academic pediatric cardiologist. Int J Fam Med 2010: 370731.

7. Martins P. Sopro Cardíaco. In: Conceitos Básicos de Cardiologia Pediátrica. Lisboa: Mar da Palavra-Edições; 2009.

8. Alvares S, Ferreira M, Ferreira H, Mota CR. Avaliação inicial do sopro cardíaco na criança: papel dos exams complementares.Rev Port Cardiol 1997 Jul-Ago; 16 (7-8): 621-4, 588; 625.

9. Saunders NR. Innocent heart murmurs in children: taking a diagnostic approach. Can Fam Physician 1995 Sep; 41: 1507-12.

10. Kwiatkowski D, Wang Y, Cnota J. The utility of outpatient echocardiography for evaluation of asymptomatic murmurs in children. Congenit Heart Dis 2012 May-Jun; 7 (3): 283-8.

\section{CONFLITOS DE INTERESSE}

As autoras declaram não ter conflito de interesses.

\author{
ENDEREÇO PARA CORRESPONDÊNCIA \\ Rita S. Oliveira \\ Largo Poeta Ary dos Santos, $\mathrm{n}^{\circ} 1-5^{\circ} \mathrm{A}$ \\ Alfornelos - 2700-662 Amadora \\ e-mail: ritas-oliveira@hotmail.com
}

Recebido em 03-07-2013

Aceite para publicação em 18-12-2013

\section{ABSTRACT}

\section{PAEDIATRIC HEART MURMUR: A CASE SERIES STUDY}

Introduction: Heart murmurs are detected in many children. They are a common cause of referral and it is important to be aware of the warning signs for significant murmurs when evaluating a child with a heart murmur.

Purpose: To analyze the population referred to a Pediatric Cardiology Department with a cardiac murmur.

Material and Methods: This is a study of a case series of children referred for investigation of a cardiac murmur. The authors reviewed the cases referred between January to March 2012 with the diagnosis of heart murmur, assessing demographic data, clinical history, physical findings, and the results of laboratory tests, including transthoracic echocardiography and electrocardiography.

Results: During the study period, there were a total of 743 first consultations. In 197 children (26.5\%) a heart murmur was the reason for referral. Of the sample, 51.3\% were male and the majority (about 70\%) of patients were younger than 2 years. In almost all of the children the murmur was described as innocent and there were no other significant finding on physical examination. In about one third of cases, anomalies were found with echocardiographic assessment. There was a significant association between a non-innocent murmur and abnormal findings on physical examination and echocardiography. There was also a significant association between important murmurs and age less than one year with ultrasound findings.

Discussion: Cardiac murmurs in children are a frequent reason for referral. Most murmurs in children are benign. Clinical features allow for differentiation between innocent and pathological murmurs. As described in previous studies, abnormalities found on physical examination and younger ages are warning signs that require a more detailed evaluation.

Key-words: Heart Murmur; Children. 